\title{
René Zazzo \\ 1910-1995
}

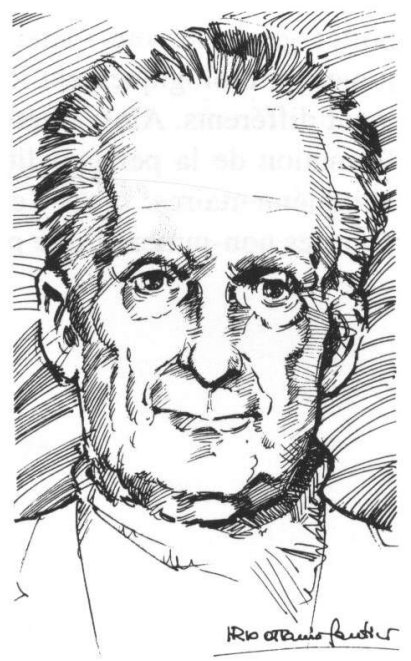

René Zazzo, né en 1910 fut une des brillantes figures de la psychologie; il vient de nous quitter, en ce 20 septembre 1995 et son départ laisse un grand vide pour tous ceux qui l'ont connu et côtoyé. Pionnier de l'étude du développement de l'enfant, il a marqué la psychologie par ses travaux sur les jumeaux, la reconnaissance de soi, l'attachement et l'intelligence. Collaborateur dès 1936 du Professeur Henri Wallon et son successeur en 1950 à la direction du Laboratoire de psycho-biologie de l'enfant (Ecole pratique des Hautes Etudes), René Razzo s'employa tout au long de sa carrière à coordonner pratique (clinique, pedagogie) et recherche fondamentale. Universitaire engagé, il a été un homme de terrain qui a lutté au-delà des chapelles, contre le divorce des disciplines sans pour autant céder à une forme illusoire d'unité de la psychologie. Entre 1955 et 1977, il fut président de la Société française de psychologie.

En 1933, il achève des études de philosophie à la Sorbonne puis quitte la France pour les Etats-Unis. A Yale, Gesell l'accueille dans son Laboratoire et l'initie à son approche. L'intérêt porté, non pas à l'hérédité et au milieu dans l'expression des différences individuelles, mais bien plus aux relations existant entre les processus de maturation et apprentissages, vont marquer René Zazzo et influencer en partie son orientation future. L'autre figure qui influencera ses perspectives est la rencontre avec Henri Wallon dès 1936.

Les trois institutions majeures au sein desquelles il a exercé puis mené ses recherches et ses enseignements ont été respectivement: le Laboratoire de Psycho-biologie de l'école Pratique des Hautes Etudes, le Laboratoire de Psychologie de l'hôpital Henri Rousselle (Hôpital Sainte Anne, Paris, France) qu'il a dirigé de 1940 à 1980 et la Section de Psychologie génétique et de psychologie de l'Enfant de l'Université de Paris X-Nanterre (France), section qu'il a créée et dirigée de 1967 a 1980.

Dans tous les cas, il a développé le travail en équipe, sa recherche individuelle venant à tout moment se greffer sur le travail collectif et l'enrichir.

René Zazzo a aussi été un enseignant exceptionnel, tant à l'Université, dans son Laboratoire, qu'à l'hôpital. Ses laboratoires, son enseignement, ont toujours été ouverts à 
des personnes venant d'ailleurs, d'autres pays ou d'autres disciplines, permettant ainsi une grande ouverture d'esprit et un enrichissement réciproque des participants.

Ses recherches personnelles sur les jumeaux se présentent comme une méthode de portée générale pour l'analyse des processus d'individuation. Il met en évidence que les jumeaux, biologiquement identiques et élevés dans le même milieu, sont psychologiquement différents. Ainsi l'hérédité et les facteurs de milieu n'expliquent pas à eux seuls la formation de la personnalité. La vie en commun donne aux deux partenaires des rôles complémentaires: c'est l'effet-de-couple, révélé par les jumeaux, qui joue aussi bien pour les non-jumeaux que pour n'importe quel couple.

C. Droehnle-Breit 